\title{
Sagittal Balance, Pulmonary Function, and Spinopelvic Parameters in Severe Post-Tubercular Thoracic Kyphosis
}

\author{
Pratyush Shahi, Manish Chadha, Apoorv Sehgal, Aarushi Sudan, \\ Umesh Meena, Kuldeep Bansal, Dheeraj Batheja \\ Department of Orthopaedics, University College of Medical Sciences, New Delhi, Delhi, India
}

\begin{abstract}
Study Design: Cross-sectional study.
Purpose: To evaluate sagittal balance, pulmonary function, and spinopelvic parameters in patients with healed spinal tuberculosis with severe thoracic kyphosis.

Overview of Literature: Deterioration of neurological function is an absolute indication of surgical intervention in severe posttubercular kyphosis, but the relationship of compromise in lung function and spinal alignment with severity of kyphosis is still unclear. Methods: Twenty patients (age, 14-60 years) with healed spinal tuberculosis with thoracic kyphosis $>50^{\circ}$ were included. Lateralview radiography of the whole spine, including both hips, was performed for assessment of kyphotic angle (K angle), sagittal balance, lumbar lordosis, and spinopelvic parameters. Pulmonary function was assessed by measuring the forced vital capacity (FVC), forced expiratory volume in 1 second (FEV1), and their ratio (FEV1/FVC) by spirometry.

Results: A positive correlation between severity of kyphosis and sagittal imbalance was noted, with compensatory mechanisms maintaining the sagittal balance in only up to $80^{\circ}$ of dorsal kyphosis. In $>80^{\circ}$ of kyphosis, FVC was found to be markedly decreased (mean $\mathrm{FVC}=50.6 \%$ ). The mean $\mathrm{K}$ angle was lower in subjects with lower thoracic kyphosis. In lower thoracic kyphosis, due to short Iordotic and long kyphotic curves, both lumbar lordosis and pelvic retroversion worked at compensation, whereas, in middle thoracic kyphosis, due to long lordotic curve, only lumbar lordosis was required. Normal pulmonary function (mean FVC, 83.0\%) and lesser kyphotic deformity (mean $\mathrm{K}$ angle in adolescents, $69.8^{\circ}$; in adults, $94.4^{\circ}$ ) were found in adolescents.

Conclusions: In $>80^{\circ}$ of thoracic kyphosis, there is sagittal imbalance and a markedly affected pulmonary function. Such patients should be offered corrective surgery if they are symptomatic and medically fit to undergo the procedure. However, whether the surgical procedure would result in improved pulmonary function and sagittal balance needs to be evaluated by a follow-up study.
\end{abstract}

Keywords: Thoracic spine; Post-tubercular kyphosis; Sagittal balance; Pulmonary function; Spinopelvic parameters

\section{Introduction}

Tuberculosis of the spine is a common spinal pathology in India. It accounts for approximately half of all cases of musculoskeletal tuberculosis. Characteristically, there is diminution in the intervertebral disc space and ad-

Received Sep 3, 2020; Revised Nov 30, 2020; Accepted Dec 8, 2020

Corresponding author: Pratyush Shahi

Department of Orthopaedics, University College of Medical Sciences, Dilshad Garden, New Delhi, Delhi-110095, India

Tel: +91-9560484211, E-mail: pratyushshahi@gmail.com 
jacent vertebral bodies, collapse of the spinal elements, and anterior wedging, leading to kyphosis and gibbus formation. Antitubercular chemotherapy remains the cornerstone of treatment [1], but $3 \%$ of patients treated conservatively will develop kyphotic deformity of $>50^{\circ}$, which can cause serious cosmetic, psychological, cardiorespiratory, and neurological problems [2].

Currently, the indications cited by various studies in literature for vertebral body resection and deformity correction in severe post-tubercular kyphosis include progressive neural deficit (i.e., late-onset paraplegia), compromised lung function, and altered sagittal balance $[3,4]$. Although it is obvious that deterioration in neurological function should be an indication for surgical intervention, the relationship of compromise in lung function and spinal alignment with severity of kyphosis is still unclear. Moreover, recent studies suggest that there is no significant improvement in lung function following vertebral column resection and deformity correction [5]. Since compensatory lordosis of the lumbar spine results from focal post-tubercular kyphosis, there may not be a significant sagittal plane imbalance in such patients. Hence, these two factors, in absence of neural deficit, may not warrant surgical correction as the associated morbidity and mortality with such intervention is high.

Our study aimed to assess pulmonary function and sagittal balance in patients with healed post-tubercular kyphosis of $>50^{\circ}$ and evaluate if they are significantly affected to be an indication for deformity correction in absence of neural deficit.

\section{Materials and Methods}

This cross-sectional study included 20 patients aged 14-60 years with healed spinal tuberculosis with thoracic kyphosis $>50^{\circ}$. The study obtained ethical approval of the institutional review board of University College of Medical Sciences, Delhi, India on October 15, 2017 (registration no, IEC-HR/2017/32/78). Informed consent was obtained from all individual participants included in the study.

Lateral-view radiography of the whole spine, including both hips, with the patient standing erect and both hips and knees in complete extension to negate the compensatory mechanisms at the hips and knees, was performed to assess sagittal balance, kyphotic deformity, and spinopelvic parameters.

Kyphotic angle ( $\mathrm{K}$ angle) was measured by drawing a

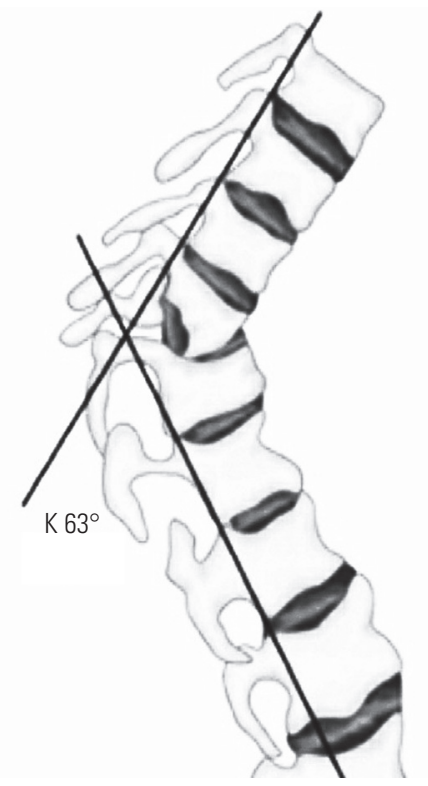

Fig. 1. Measurement of kyphotic angle (K angle).

line along the posterior margins of the healthy vertebrae above and below the site of the disease. The angle formed by the two lines is the K angle (Fig. 1) [6].

Sagittal balance was measured by drawing a vertical line (plumb line) from the middle of the body of the $\mathrm{C} 7$ vertebral body. This line should normally pass through the superior endplate of $\mathrm{S} 1$ or more precisely within $2 \mathrm{~cm}$ of the posterosuperior corner of the S1 vertebral body. Then, the position of this line was termed positive $(>2 \mathrm{~cm}$ anterior to the posterosuperior corner of S1), neutral (within $2 \mathrm{~cm}$ from the posterosuperior corner of S1), or negative ( $>2$ $\mathrm{cm}$ posterior to the posterosuperior corner of S1) (Fig. 2) [7].

Pulmonary function was assessed by measuring the forced vital capacity (FVC), forced expiratory volume in 1 second (FEV1), and their ratio by spirometry and comparing the values in the form of percentage to the predicted values. Posteroanterior view chest radiography was performed in all cases to rule out gross pulmonary lesions, and such patients were excluded from the study to ensure the measured deterioration in lung function was only due to extrapulmonary restrictive lung disease secondary to kyphotic deformity.

Spinopelvic parameters (pelvic incidence [PI], pelvic tilt $[\mathrm{PT}]$, and sacral slope [SS]) were measured on the whole spine lateral-view radiography, including both hips. PI is the angle between the line perpendicular to the sacral 


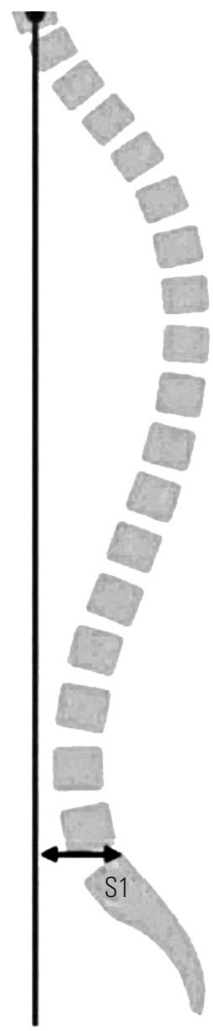

Positive

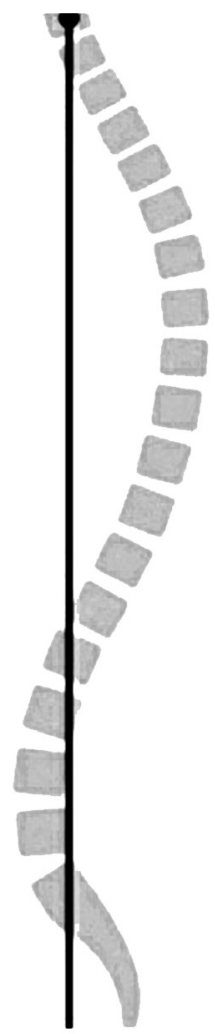

Neutral

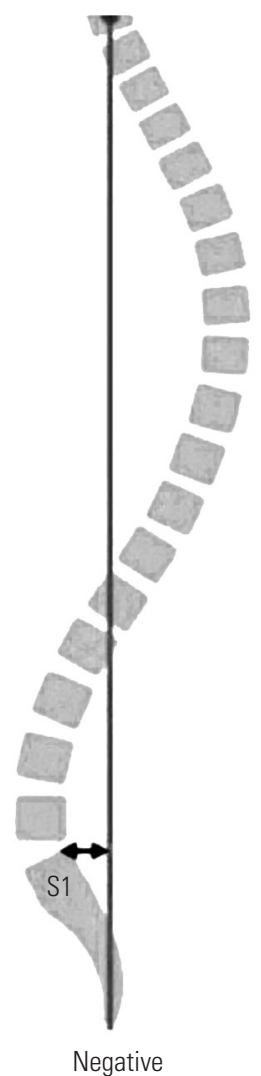

Negative
Fig. 2. Sagittal balance

plate at its midpoint and the line connecting this point to the axis of rotation of the femoral head. PT is the angle between the line connecting the midpoint of the superior sacral end plate to the femoral rotational axis and the line extending vertically from the femoral rotational axis. SS is the angle between the superior endplate of $S 1$ and a horizontal line extending from the anterior-inferior corner of the S1 endplate (Fig. 3) [8].

Lumbar lordosis (LL) was determined based on the angle between the superior end plate of L1 and superior end plate of S1 (Cobb's L1-S1 method) [9].

\section{Results}

The mean age of the patients was 25.8 years (range, $14-50$ years). Six of 20 patients (30\%) were adolescents (14 to 18 years), and 14 (70\%) were adults. Twelve patients $(60 \%)$ were female, and 8 (40\%) were male.

The mean $\mathrm{K}$ angle was $87.1^{\circ}$ (range, $60^{\circ}-140^{\circ}$ ). Eleven of 20 patients (55\%) maintained sagittal balance, while the remaining $9(45 \%)$ had sagittal imbalance. A majority of patients had decreased FVC (17/20), a marked decrease

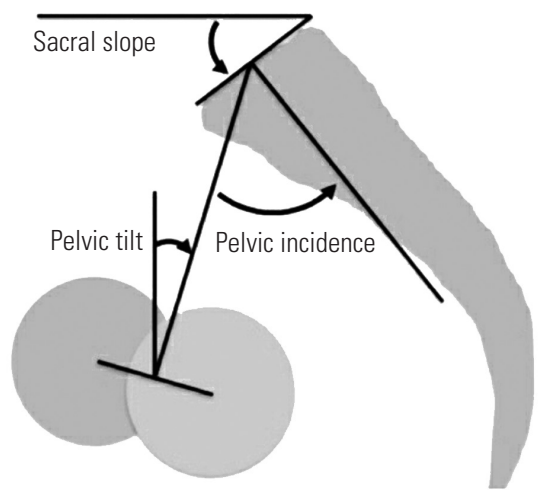

Fig. 3. Spinopelvic parameters.

Table 1. Kyphotic angle and FVC

\begin{tabular}{lcc} 
Kyphotic angle & No. of patients $(\%)$ & Mean \% FVC \\
$60^{\circ}-80^{\circ}$ & $11(55)$ & 73.3 \\
$81^{\circ}-100^{\circ}$ & $3(15)$ & 59.8 \\
$101^{\circ}-120^{\circ}$ & $3(15)$ & 56 \\
$121^{\circ}-140^{\circ}$ & $3(15)$ & 36.3 \\
\hline
\end{tabular}

FVC measured as percentage of predicted value.

FVC, forced vital capacity.

being noted in patients with $\mathrm{K}$ angle $>80^{\circ}$ (Table 1 ). $\mathrm{K}$ angle was found to be significantly lower in adolescents (mean, 69.8 $8^{\circ}$ than adults (mean, $94.4^{\circ}$ ). FVC was found to be significantly reduced in adults (mean, $54.4 \%$ of predicted) compared to that in adolescents (mean, $83.0 \%$ of predicted). Fifteen of 20 subjects (75\%) had the apex of deformity in the lower thoracic (at or below T9) level, and these subjects had lower mean $\mathrm{K}$ angle, lower mean LL, and higher mean PT/PI ratio compared to subjects with middle thoracic deformity (T4-T8) (Table 2).

Sagittal alignment showed dependence on K angle, LL angle, and PT/PI ratio but not on PI. The mean FVC was markedly reduced in patients with uncompensated sagittal alignment (Table 3). Pathological PT/PI ratio $(>0.5)$ and a lesser mean LL angle $\left(65^{\circ}\right)$ were noted in patients with PI $<35^{\circ}$ (Table 4).

\section{Discussion}

Spinal tuberculosis can lead to severe kyphotic deformity of the back, which can further cause progressive neural deficit, decrease in lung function, and spinal malalignment. Although deterioration in neural status is an absolute indication for corrective surgery, a consensus has not been reached if the latter two, in absence of neural deficit, 
Table 2. Apex of deformity

\begin{tabular}{|c|c|c|c|c|c|c|}
\hline Level & No. of subjects & Patients with compensated alignment (\%) & Mean $\mathrm{K}$ angle $\left({ }^{\circ}\right)$ & Mean LL $\left({ }^{\circ}\right)$ & Mean PT/PI & Mean SS $\left(^{\circ}\right)$ \\
\hline Lower (T9 and below) & 15 & $9(60)$ & 82.2 & 75.6 & 0.40 & 25.9 \\
\hline Middle (T4-T8) & 4 & $1(25)$ & 108.5 & 86.8 & 0.19 & 46.5 \\
\hline Upper (T3 and above) & 1 & $1(100)$ & 63.0 & 50.0 & 0.30 & 30.0 \\
\hline
\end{tabular}

K angle, kyphotic angle; LL, lumbar lordosis; PT, pelvic tilt; PI, pelvic incidence; SS, sacral slope.

Table 3. Sagittal alignment—compensated and uncompensated

\begin{tabular}{lccccc} 
Sagittal alignment & No. of patients & Mean K angle $\left({ }^{\circ}\right)$ & Mean LL $\left(^{\circ}\right)$ & Mean PI $\left({ }^{\circ}\right)$ & Mean PT/PI \\
Compensated & 11 & 77.1 & 71.9 & 44.6 & 0.30 \\
Uncompensated & 9 & 99.4 & 82.2 & 40.3 & 51.1 \\
\hline
\end{tabular}

FVC measured as percentage of predicted.

K angle, kyphotic angle; LL, lumbar lordosis; PI, pelvic incidence; PT, pelvic tilt; FVC, forced vital capacity.

Table 4. Relation of PI with PT/PI ratio, PT, and LL

\begin{tabular}{lcccc}
\hline $\mathrm{PI}\left({ }^{\circ}\right)$ & No. of patients $(\%)$ & Mean PT/PI & Mean PT & Mean LL \\
\hline $20-35$ & $4(20)$ & 0.58 & 14.2 & 65.0 \\
\hline $36-50$ & $12(60)$ & 0.29 & 12.5 & 80.2 \\
\hline $51-70$ & $4(20)$ & 0.30 & 17.5 & 80.5 \\
\hline
\end{tabular}

PI, pelvic incidence; PT, pelvic tilt; LL, lumbar lordosis.

should also warrant surgery.

Various mechanisms from top to bottom, namely, lumbar hyperlordosis, pelvic retroversion, hip extension, knee flexion, and ankle extension, act to compensate for the thoracic kyphotic deformity and maintain sagittal balance. When the energy required to sustain these compensatory mechanisms becomes high, the patient can turn symptomatic and present with pain and disability. Eventually, the spinal deformity can surpass these compensations, obviating the need for an external support.

In this study, we noted a strong correlation between spinal alignment and severity of kyphosis. The mean $\mathrm{K}$ angle of kyphosis was $77.1^{\circ}$ in patients with sagittal balance and $99.4^{\circ}$ in the patients with imbalance, indicating that, at up to $80^{\circ}$ of dorsal kyphosis, the compensatory mechanisms are able to maintain the sagittal balance. To the best of our knowledge, no previous study has been conducted to determine the relationship between severity of posttubercular kyphosis and spinal alignment.

Kyphosis leads to restrictive lung disease by altering the shape and size of the thoracic cavity, reducing its mobility and causing thoracic muscle weakness. In our study, a strong correlation was noted between severity of kyphosis and compromise in lung function. FVC was significantly reduced, signifying restrictive lung disease, in patients with kyphotic angle of $>80^{\circ}$. Although various studies correlating kyphosis and lung function have been conducted with varying results, none have been conducted on posttubercular kyphotic subjects specifically [10-14].

Moreover, $75 \%$ of the subjects had lower thoracic kyphotic deformity, which is obvious as this is the spinal region most commonly affected by tuberculosis [1]. Subjects with lower dorsal deformity had lower mean $\mathrm{K}$ angle $\left(82.2^{\circ}\right)$ compared to those with middle thoracic deformity $\left(108.5^{\circ}\right)$. Lesser deformity, when the apex of deformity was in the lower thoracic region, led to larger percentage of subjects (60\% versus $25 \%$ ) with compensated spinal alignment in this subgroup. The level of apex of the kyphotic deformity was also used to decide as to which compensatory mechanism will play the major role. In lower dorsal kyphosis (T9 and below), due to a short lordotic and long kyphotic curve, both LL and pelvic retroversion worked at compensation, whereas, in middle dorsal kyphosis (T4-T8), due to a long lordotic curve with more vertebral bodies, only LL and not pelvic retroversion was required (Figs. 4, 5). This inference was drawn from the classification system of spinal alignment proposed by Roussouly and Nnadi [15], which was based on the thoracolumbar split (as a measure of the length ratio of thoracic and lumbar curves). Lamartina et al. [16] had drawn a similar correlation between deformity pattern and compensatory mechanism. No correlation was found between the level of apex of deformity and compromise of lung function. 

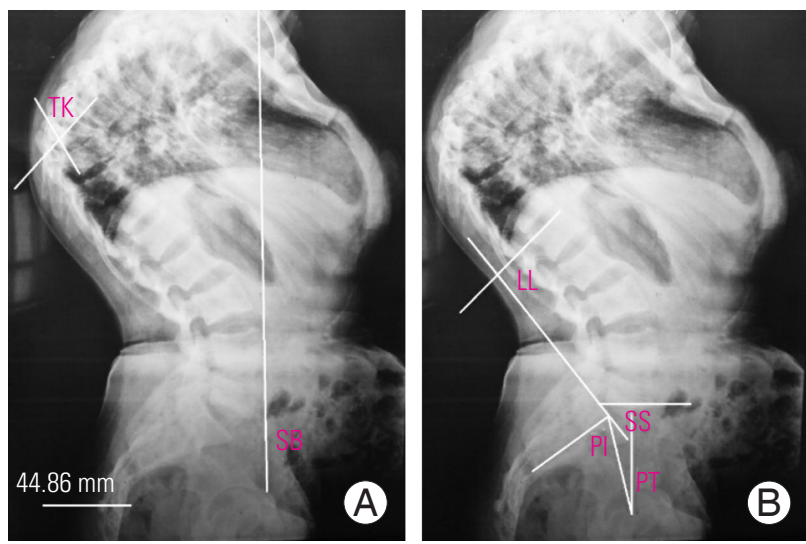

Fig. 4. (A, B) A 25-year-old female with mid-dorsal kyphotic deformity of $84^{\circ}$. Apex of deformity was in the mid-dorsal region and lumbar lordosis of $96^{\circ}$ was the only compensatory mechanism. With a pelvic tilt (PT)/pelvic incidence (PI) ratio of 0.23 and sacral slope (SS) of $50^{\circ}$, there was no pelvic retroversion compensating for the deformity. Despite the exaggerated lumbar lordosis (LL), the sagittal balance (SB) is positive. TK, thoracic kyphosis.
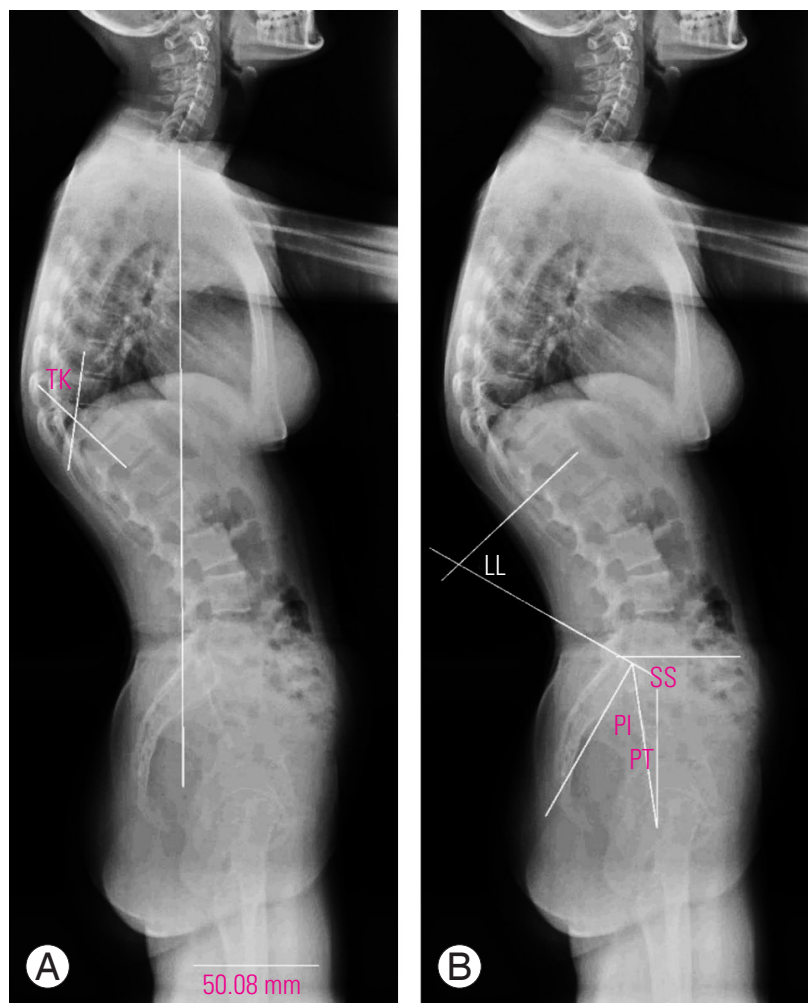

Fig. 5. (A, B) A 15-year-old female with lower thoracic kyphosis (TK) of $62^{\circ}$. Here, only lumbar lordosis $\left(\mathrm{LL}, 80^{\circ}\right)$ was enough to maintain the spinal alignment and pelvic retroversion (pelvic tilt [PT]/pelvic incidence [PI] ratio of 0.35 and sacral slope $[S S]$ of $33^{\circ}$ ) was not required.

$\mathrm{K}$ angle was found to be significantly lesser in adolescents (age, 14-18 years) than in adults (age, 19-60 years). Rajasekaran [17] had previously stated that post-tuber- cular deformity progresses in two distinct phases: phase I (active phase) includes the changes in the first 18 months during the period of activity, and phase II (healed phase) occurs after the healing of the disease. Adults have a lesser deformity at presentation, lesser increase in deformity during phase I, and virtually no change after the disease has healed. The progression of deformity in adults is usually $<30^{\circ}$ and restricted to the first phase. In contrast, children have a higher degree of deformity at presentation, a greater tendency for collapse during the active phase of the disease, and continued and variable progression even after the disease is treated and growth is complete [17]. However, we cannot categorically state this due to our limitation of the inability to follow the patient to observe for any progression, this being a cross-sectional study. The mean FVC was found to be normal in adolescents (83\%), which is relatable to their less extent of kyphotic deformity and the conclusion of Zeng et al. [18] that younger (flexible) patients are more likely to have improved function than older (ankylosed) patients following corrective surgery for kyphosis.

We studied the spinopelvic relationship by analyzing the pelvic parameters, namely, PI, PT, and SS. PI is a fixed, angular parameter and reflects the sagittal length of the pelvis from the sacral endplate to the center of the hips. It is an anatomical constant for an individual and cannot be modified after skeletal maturity, unless trauma or surgery modifies the shape of the pelvis. Ranging from $30^{\circ}$ to $80^{\circ}$, its value does not provide a good clinical correlate [16]. The mean PI in our study was $42.7^{\circ}$, which is in agreement with the observation of Moon et al. [19] that mean PIs in Asian individuals are generally lower than in Caucasian individuals. Whereas pelvic sagittal morphology is defined by PI, pelvic orientation in the sagittal plane, based on the amount of forward or backward rotation of the pelvis around the hip joints, is denoted by PT. A higher PT signifies a more retroverted pelvis, whereas a lower PT means that the pelvis is anteverted. An anteverted pelvis is energy saving because it brings the loading axis of the trunk on the pelvis (through the sacral endplate) closer to the loading axis of the pelvis on the lower limbs (through the hip joints) [20]. Conversely, pelvic retroversion causes a more horizontal sacral endplate and a more posterior basement of the spine, thus helping to compensate for a forward displacement of the spine. It is an energy-consuming compensatory mechanism for imperfect sagittal balance [16]. SS is a measure of pelvic orientation. Both 


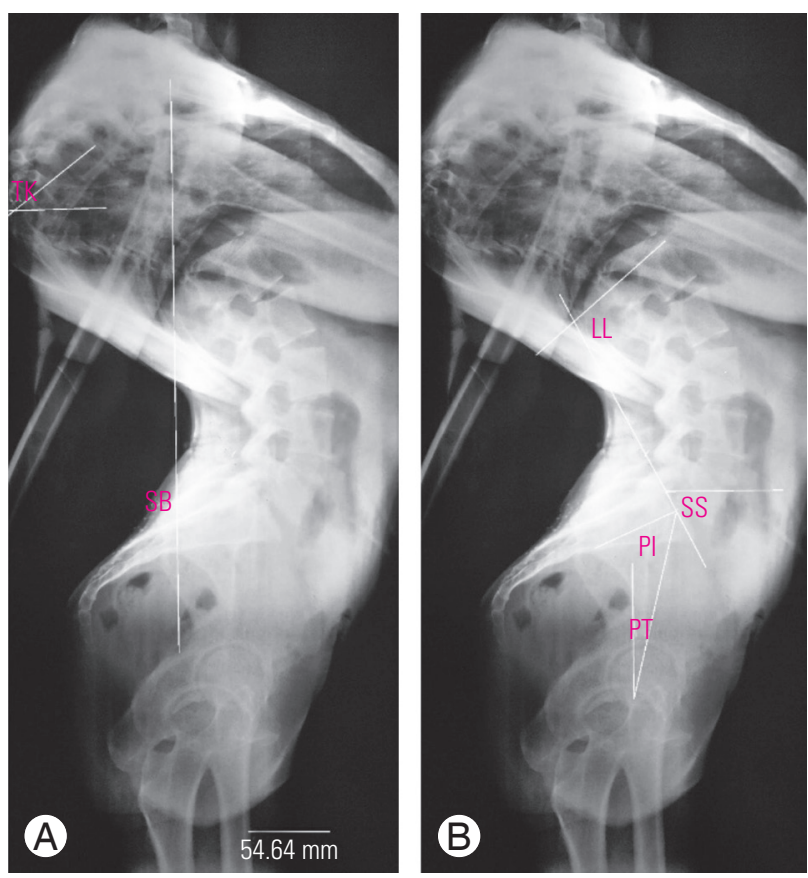

Fig. 6. (A, B) A 20-year-old female with mid-dorsal kyphosis of $140^{\circ}$. Here, longer lordotic curve allowed exaggerated lumbar lordosis (LL) of $100^{\circ}$. This led to an almost horizontal sacrum with inability to retrovert the pelvis (pelvic tilt $[\mathrm{PT}] /$ pelvic incidence $[\mathrm{PI}]$ is 0.33 and sacral slope [SS] is $\left.60^{\circ}\right)$. PT was negative $\left(-15^{\circ}\right)$ as femoral heads were behind the sacrum. So, here, due to hyperlordosis with anteverted pelvis and severe degree of deformity, the C7 plumb line had actually fallen behind the body, causing a negative sagittal balance (SB).

PT and SS are postural parameters. Their mean values in our study were $14.4^{\circ}$ and $29.8^{\circ}$, respectively.

An established arithmetic equation states that $\mathrm{PI}=\mathrm{PT}+\mathrm{SS}$. This makes PT dependent on PI; thus, the absolute value of PT is not a good clinical correlate. Mac-Thiong et al. [21] proposed that PT should be $<50 \%$ of PI for pelvic retroversion, as a compensatory mechanism, to be nonpathological. In our study, in four patients who had PI $<35^{\circ}$, the mean PT/PI ratio was 0.58 , whereas it was 0.30 in patients with $\mathrm{PI}>35^{\circ}$. This emphasizes two facts in subjects with less PI: one that the individual's ability to retrovert the pelvis will be less, and two that as it will still be used as a compensatory mechanism, this pelvic retroversion will inadvertently fall in the pathological range, causing symptoms to the subject. We concluded that a higher PI provides an individual more ability to compensate for severe thoracic kyphotic deformity in an energy-efficient way.

A positive uncompensated or neutral compensated sagittal balance is expected in patients with severe kyphosis, but we had two patients in our study with negative sagittal balance. It was noted that both of these had very severe kyphosis $\left(140^{\circ}\right.$ and $\left.130^{\circ}\right)$ and both had the apex of deformity in the mid-dorsal region (D5-D8). Mid-dorsal kyphosis provided a relatively longer lordotic curve, allowing exaggerated lordosis, which led to a more horizontal sacrum and also the inability to retrovert the pelvis. This hyperlordosis with anteverted pelvis caused the $\mathrm{C} 7$ plumb line to fall behind the body, leading to a negative sagittal balance (Fig. 6).

The limitations of the study include its cross-sectional design (thus the inability to follow the adolescent subjects to monitor for deformity progression) and less sample size.

\section{Conclusions}

In $>80^{\circ}$ of thoracic kyphosis, the subjects tend to have sagittal imbalance and markedly affected pulmonary function. Such patients should be offered corrective surgery if they are symptomatic and medically fit to undergo the procedure. However, whether the surgical procedure would result in improved pulmonary function and sagittal balance and functionality needs to be evaluated by a follow-up study.

\section{Conflict of Interest}

No potential conflict of interest relevant to this article was reported.

\section{Author Contributions}

PS and MC designed the study protocol, conducted the research, and wrote the final version of the manuscript. ApS, AaS, UM, KB and DB did the literature search, collected the data, interpreted the results, and drafted the manuscript.

\section{References}

1. Garg RK, Somvanshi DS. Spinal tuberculosis: a review. J Spinal Cord Med 2011;34:440-54.

2. Rajasekaran S. Kyphotic deformity in spinal tuberculosis and its management. Int Orthop 2012;36:359-65.

3. Jain AK, Dhammi IK, Jain S, Mishra P. Kyphosis in spinal tuberculosis: prevention and correction. Indian J Orthop 2010;44:127-36.

4. Cavanilles-Walker JM, Ballestero C, Iborra M, Ubi- 
erna MT, Tomasi SO. Adult spinal deformity: sagittal imbalance. Int J Orthop 2014;1:64-72.

5. Bumpass DB, Lenke LG, Bridwell KH, et al. Pulmonary function improvement after vertebral column resection for severe spinal deformity. Spine (Phila Pa 1976) 2014;39:587-95.

6. Tuli SM. Severe kyphotic deformity in tuberculosis of the spine. Int Orthop 1995;19:327-31.

7. Cheung JPY. The importance of sagittal balance in adult scoliosis surgery. Ann Transl Med 2020;8:35.

8. Celestre PC, Dimar JR 2nd, Glassman SD. Spinopelvic parameters: lumbar lordosis, pelvic incidence, pelvic tilt, and sacral slope: what does a spine surgeon need to know to plan a lumbar deformity correction? Neurosurg Clin N Am 2018;29:323-9.

9. Vacari DA, Neves EB, Ulbricht L. Comparison between methods of assessing lumbosacral curve obtained by radiographic image. Acta Ortop Bras 2015;23:67-71.

10. Abbi G, Lonner BS, Toombs CS, et al. Preoperative pulmonary function in patients with operative scheuermann kyphosis. Spine Deform 2014;2:70-5.

11. Kanathur N, Lee-Chiong T. Pulmonary manifestations of ankylosing spondylitis. Clin Chest Med 2010;31:547-54.

12. Sampaio-Barros PD, Cerqueira EM, Rezende SM, et al. Pulmonary involvement in ankylosing spondylitis. Clin Rheumatol 2007;26:225-30.

13. Harrison RA, Siminoski K, Vethanayagam D, Majumdar SR. Osteoporosis-related kyphosis and impairments in pulmonary function: a systematic review. J Bone Miner Res 2007;22:447-57.
14. Horie J, Murata S, Inoue Y, et al. A study of the influence of the pulmonary function on the angles of thoracic kyphosis and lumbar lordosis in communitydwelling elderly women. J Phys Ther Sci 2009;21:16972.

15. Roussouly P, Nnadi C. Sagittal plane deformity: an overview of interpretation and management. Eur Spine J 2010;19:1824-36.

16. Lamartina C, Berjano P, Petruzzi M, et al. Criteria to restore the sagittal balance in deformity and degenerative spondylolisthesis. Eur Spine J 2012;21(Suppl 1):S27-31.

17. Rajasekaran S. Natural history of Pott's kyphosis. Eur Spine J 2013;22(Suppl 4):634-40.

18. Zeng Y, Chen Z, Ma D, et al. The influence of kyphosis correction surgery on pulmonary function and thoracic volume. Spine (Phila Pa 1976) 2014;39:177784.

19. Moon JW, Shinn JK, Ryu D, Oh SY, Shim YS, Yoon $\mathrm{SH}$. Pelvic incidence can be changed not only by age and sex, but also by posture used during imaging. Korean J Spine 2017;14:77-83.

20. Boulay C, Tardieu C, Hecquet J, et al. Sagittal alignment of spine and pelvis regulated by pelvic incidence: standard values and prediction of lordosis. Eur Spine J 2006;15:415-22.

21. Mac-Thiong JM, Roussouly P, Berthonnaud E, Guigui P. Sagittal parameters of global spinal balance: normative values from a prospective cohort of seven hundred nine Caucasian asymptomatic adults. Spine (Phila Pa 1976) 2010;35:E1193-8. 